\title{
INTRODUCTION
}

\section{(Super) Diversities \& HIV/AIDS: Multilingualism in Multiple Modalities}

\author{
Nobuhle Luphondo \\ Department of Linguistics, University of the Western Cape, Private Bag X17, Bellville 7535, South Africa \\ E-mail: nluphondo@uwc.ac.za \\ Christopher Stroud \\ Department of Linguistics, University of the Western Cape, Private Bag X17, Bellville 7535, South Africa/ \\ Centre for Research on Bilingualism, Stockholm University, S-106 91 Stockholm \\ E-mail: cstroud@uwc.ac.za; Christopher.Stroud@biling.su.se
}

We live in a multilingual and multi-discursive context, in which meanings and identities are negotiated and renegotiated in multiple ways and across multiple seams, in an environment that is characterized by great linguistic and discursive heterogeneity. An increasing multidirectionality of migration/mobility has replaced traditional forms of in-out migration; new modes of digital and mobile communication have distributed the presence and identities of individuals across multiple, virtual and 'real' co-temporaneous spaces; an accelerating diversification and growth of global markets and technologies are creating complex zones of contact and negotiation. In the postcolonial contexts of the South, a third dimension of diversity - temporality - the outcome of new vertical and horizontal mobilities of historically separated populations, and social transformation is complexifying the idea of modernity/late modernity. The notion of 'superdiversity' (Vertovec 2007, 2010) captures the impact that the scale and multidimensional nature of mobility - demographic, sociocultural, spatial, technological, material, economic and metaphysical - is having on the conditions of human existence in the $21^{\text {st }}$ Century.

Coping with diversity on this scale thus requires new modes of theoretical engagement and new approaches to how we engage discursively with, represent, and enact co-existence and conflict in contexts of extreme social transformation, mindful that discourses of diversity and equality cannot be separated (Blommaert and Verschueren 1998). At the same time, theorizing diversity must engage with its multiple manifestations, local representations and practices. This is particularly the case for the Southern African context with its recent history of postcoloniality. In the African context in particular, health care sector diversity in terms of language, culture, race, religion and major socioeconomic differences are extensive. Language and communication practices are central to any understanding of HIV/AIDS discourses, and they are among the most sensitive indicators, as well as mediators of, diversity. However, diversity is often erased in favour of the uniform and the conformist, and language is one of the key instruments in the dynamics that makes disguising and obfuscating 
diversity possible. Thus, this special issue of SPIL PLUS is devoted to diversity in the linguistic representation, mediation and negotiation of HIV/AIDS discourses in their many local contexts. All papers focus here on how insights from a re-thinking of language from the vantage point of diversity can contribute to understanding the local and highly specific manifestations of HIV/AIDS, more specifically to understanding how diversity in language and communication can be harnessed to the benefit of diagnosis, treatment and social awareness HIV/AIDS.

Tommaso Milani's paper addresses how constructions of uniformity take place around everyday discourses of health and HIV/AIDS. Milani asks (1) why some forms of categorization become naturalized and thus made commonsensical at different moments in different contexts and (2) what kinds of real effects these natural categories have on people in their daily lives. In particular he takes issue with the notion of identity, a seemingly innocuous idea that we use to orientate our lives around in all manner of contexts. He takes a queer theory approach to sexuality discourses, a powerful theoretical tool for problematizing "normative consolidations of sex, gender and sexuality" (Jagose 1996: 99), allowing "the problems and leakages of identity categories" to be articulated (Yep 2003:39). Milani's Critical Discourse Analysis of two medical interactions, interpreted within the 'matrix of intelligibility' reveals the mechanisms behind how desires and practices are interpreted as "naturally coextensive with particular sexual identities"; having sex with men, for example, automatically becomes indicative of belonging to the category homosexual, irrespective of what other desires (e.g. desires for loving women) the person may hold. From this attribution of category, contention and scapegoating follow, as do, even more problematically, inappropriately conceived interventions, such as focusing on risk groups rather than attending to practices and particular discourses. Milani also shows that even attempts to uncouple sexual practices from identities and desires, may nevertheless have the unfortunate consequence of re-affirming the categories in some ways ${ }^{1}$. The point he makes is that discourses of sexual identity need to be replaced by new discourses of desires, practices and the unconscious.

Oostendorp and Bylund in their paper share Milani's preoccupation with the pervasiveness of the seemingly commonsensical, and the dangers of slippages and the banal discriminations of the everyday. They also attend to how diversity is erased in contexts of HIV/AIDS, although their focus specifically is on the commonsense notions of language that curtail understandings of the massive cross-linguistic diversity in the encoding of emotion discourses. They note how many studies and therapy interventions around HIV/AIDS in multilingual contexts still attempt to approach HIV/AIDS carriers' emotions with the assumption that any emotion of relevance can be expressed in English and translated into other languages. This is a matrix of intelligibility that ignores the complexities of multilingualism, such as the possible lack of translation equivalents in other languages or lack of congruence in semantic fields, and the ways in which expressions of self and emotion may be differentially tied to different languages in multilingual speakers. What we could call the 'monoglossic matrix of intelligibility' also downplays problems related to language proficiencies, e.g. whether a language, say Zulu is an L1 or L2 for a speaker, the linguistic trajectories of speakers over their life-span, or how speakers stylize themselves differently across languages, varieties or styles. Although the authors do not mention it explicitly, the recent revival of the notion of

\footnotetext{
${ }^{1}$ A conundrum that we are aware of from other contexts, e.g. the apartheid racial classifications that are reaffirmed through their use in deconstructing apartheid inequities.
} 
repertoire (Blommaert and Backus 2011) or the related idea of sociolinguistic consumption (Stroud and Wee 2007, 2012) might capture better than the notion of language what it means to be a speaker in today's superdiverse contemporary world of great mobility. Both notions recognize that speakers' competencies are complex mosaics of different languages, as well as bits of language, that reflect their social and historical trajectories, and variety of groups, networks and communities, that they engage with in multilingual contexts. Thus, language resources are assembled across a wide variety of "trajectories, tactics and technologies" (Blommaert and Backus 2011), ranging from fully formal language learning to entirely informal 'encounters' with language (Stroud and Wee 2007, 2012). These different learning modes lead to different degrees of knowledge of language, from what we usually consider to be 'full and complete mastery' to simple recognition of more or less familiar linguistic elements.

Yet a third paper speaks critically to some other commonsense understandings about language and the dangers of paying too little attention to the notion of repertoire and its complex sociolinguistics. This is Elvis Saal's piece on constructed youth varieties of Afrikaans, and the commonsense notion that 'slang' or youth variety is an appropriate tool with which to reach out to youngsters with prevention information. His study is a comparison of how two groups of Afrikaans speaking youth (Coloured and White) react to HIV/AIDS prevention messages written in (a) the English Lovelife variety (what he calls 'over accommodated artificial slang', (b) two varieties of authentically constructed teenage slang (Coloured and White Afrikaans varieties, and here, each group was 'exposed' to their variety), and Standard Afrikaans. Generally, both groups expressed an aversion to the English lovelife variety, but proved to have quite distinct patterns of preference with respect to how they perceived the constructed varieties. Whereas the White Afrikaans speaking youth disliked the non-standard varieties, the group of Coloured Afrikaans speaking youth was 'comfortable' with all the varieties, saying about their variety, for example, "it's how we speak" and "it gives more meaning to the message". Interestingly, none of the groups felt that Standard Afrikaans was 'their' variety. Saal's paper reveals clearly the complexities inherent in claiming or rejecting 'ownership' of particular forms of speech, and reminds us again how complex the links are between sociolinguistic dimensions of, for example, status and solidarity, on the one hand, and the uptake and veracity accorded a message, on the other. Although it was outside the scope of his paper to delve deeper into the underlying reasons for the different evaluations of constructed youth varieties, it is tempting to reflect on the different sociopolitical and historical trajectories of each group, and the role this could have played. For example, Coloured and White Afrikaans speakers differ with respect to dynamics of language loss/purism in a changing socioeconomic context (White Afrikaans speakers) and ethnic 'identity work', recognition in normative contexts (transgressive) of new 'variety'.

In the paper by Antia and Razum, diversity is once again the theme. The paper is a comparative study of the efficacy of HIV/AIDS prevention messages in the context of Germany and Nigeria, and comprises an interesting analysis of the very different sociopolitical and cultural dimensions that frame the messages. One important dimension of the study is that it shows how a major parameter of difference behind message formation in the two contexts is that of 'fear' versus 'empowerment', where Nigerian prevention messages tend towards warning their readers of the dangers of promiscuous sexual relationships and other such practices, whereas the German messages exude the joys of sex, experimentation and personal empowerment. The authors find that each message form is tailored to its context 
and is culturally congruent, to the extent that the German sociopolitics of sexuality is very much organized around a life-style economics, with abundant access to a variety of sites of sexual production and consumption (websites, dating sites, sexual experimentation sites) with an explicit and open sexual discourse, and where the prevalence of HIV/AIDS is higher among high income, life-style groups. On the other hand, the politics and culture of sexuality in Nigeria is very different. In this context, open and explicit mention of sex is taboo, and a preponderance of problems with HIV/AIDS is typically found in low income, socially and politically marginalized groups, and among women in urban areas. In other words, major structural differences between the two contexts are reflected in the form and content of the message. Interestingly, when each of these message types are measured against an independent psychological model of message efficacy, neither type is optimally efficient, both lacking an essential mix of fear and efficacy. Antia and Razum conclude on the basis of this that, "if the theoretical acceptance profiles of the messages analyzed reflects actual audience responses, then behavioural change in the context of HIV/AIDS would have to challenge sociocultural givens and entrenched interests or ideologies that procure certain short term personal of class benefits".

Feliciano Chimbutane returns to concerns of cultural diversity in HIV/AIDS discourses with his study on the HIV/AIDS radio phone programs in Mozambique. In one sense contrary to Antia and Razum, Chimbutane suggests that failure of HIV/AIDS education campaigns may reflect problems in culturally and linguistically appropriate communication, suggesting that "[b]y framing language within its social and cultural contexts, sociolinguistically informed approaches to discourse may contribute to understanding and explaining communication failures as well as to strengthening awareness campaigns". An interesting feature of Chimbutane's work is his analysis of the grassroots' re-invention or re-appropriation of culturally embedded genres of speech in Changana to fulfil the need for new discourses and ways of talking about HIV/AIDS publically. Positioning language firmly within its local sociocultural context, Chimbutane isolates the variety of linguistic processes, ranging from semantic extensions, borrowings from Portuguese and translations found in peer talk arrangements for dealing with cultural taboos that sidestep the age and gender mixed contexts that often lead to silence or euphemism on sexual matters. As formal institutions become less important generally in late modern society, a variety of informal institutions and associative networks begin to play a larger role. What we see coming out of Chimbutane's work is a productive form of language intellectualization, sensitive to sociocultural constraints but well in keeping with the complexities of superdiverse late modernity. Chimbutane captures the diversity and complexities of the life his informants straddle in traditional cultures and modernizing societies in the following, "the two worlds are constantly relating and interpenetrating, blending and merging” (quoted from Passador 2009).

In their paper, on deconstructing gender and sexuality discourses in the "Brothers for Life" campaign, Luphondo and Stroud pick up on the theme of the constant interpenetration, blending and merging of tradition and modernity. These authors explore how multimodal representations of masculinity and sexuality (re)produce discourses about what it means to be a man. They find that these modern representations - discourses of masculinity - resonate loudly with historical ideas of maleness, and that the way masculinity is represented in health information resembles how male figures are used to advertise commercial products on an everyday basis. This paper shares many of the concerns raised by Antia and Razum about the efficacy of messages that are culturally familiar and sociopolitically congruent, (in fact, not 
surprisingly, the messages in Bassey and Razum's study with the lowest efficacy were in fact found on a poster). The paper also echoes Milani's warning that much in the everyday discourse and matrix of intelligibility serves to reproduce discourses that are both inappropriate and even consolidating of practices and attitudes that may be contrary to the goal of prevention. However, the paper also shares with Chimbutane the concern to create something new - alternative discourses and modes of representation - on the basis of the old. This requires, though, that we are able to untangle the complicated web of historical and contemporary discourses of male chauvinism embedded in every fibre of the multimodal representation. Clearly, multimodal representations with their ready affordance to involve readers in subtle and unconscious ways also speak to the question of emotion discourses raised by Oostendorp and Bylund as well as Saal. Perhaps, though, one of the most important findings of this paper, which uses Bahktin's chronotope framework, lies in the mechanics the multimodal representation uses to align the reader with the interactional regime, thus literally bringing the reader into the very construction of the representation. Readers bring with them complex sociopolitical histories and trajectories, which thereby become part of the (re)construction of the discourse. The conundrum, then, is how to tweek these biographies so that they read information differently, or to quote Antia and Razun, how to start "to challenge sociocultural givens and entrenched interests or ideologies that procure certain short term personal of class benefits".

Each of the papers works with a different modality and brings the appropriate tools to bear on the analyses of HIV/AIDS discourses in that modality. Taken as a whole, the collection thus address the bias in much work on HIV/AIDS prevention messages where communication scholars largely carry out and report on audience response studies on large-scale media rather than attend to the minute subtleties of small-scale modalities such as brochures and posters. Luphondo and Stroud talk of the need to look at chains of communication, with their suggestion that the notion of resemiotization, which follows the transport of messages across modalities and media, both linguistic and non-linguistic, offers a means of capturing how HIV/AIDS discourses might spread, and their meanings unravel and be compounded over time. In this context, they point to the productivity of the idea of affordance to capture the interaction of message with modality, that is, the different surfaces, interfaces and platforms for messages that provide different possibilities and different constraints.

Another strong feature that runs through the collection is the interdisciplinarity of the bulk of the papers. This is surely a characteristic of the epistemologies and methodologies of researching language in superdiverse contexts. Oostendorp and Bylund's paper emphasizes an original post-hoc take on interdisciplinarity, when they review earlier work on HIV/AIDS that could have invoked more attention to the obvious centrality of linguistic issues, but chose not to. Of course, HIV/AIDS has never really been defined as a linguistic problem per se, although perhaps it ought to be given the centrality of language and representation that all the papers here bear testimony to. Antia and Razum's work is clearly prototypically interdisciplinary, and they are very explicit on what a detailed linguistically informed analysis (text based, corpus linguistic, content analysis) of HIV/AIDS messages can offer the variety of other disciplines involved in HIV/AIDS communication research.

A common dimension throughout the issue shared by more or less all the authors is their ambition to highlight the important practical implications of their research. Milani's data for example, underscores the value of autobiographical data which, recognizing that there is "no 
view from nowhere, no gaze that is not positioned" (Gal \& Irvine 2000: 36) (always partial and locally situated) recognizes the empowering function of autobiographical narratives "which can form the basis of education outreach efforts from a highly personalized perspective" (Higgin and Norton 2010). Oostendorp and Bylund also emphasize (as does Milani) how recognition of diversity (especially in emotion discourses) can reinforce local strategies of HIV/AIDS treatments. Diversity is usually seen as a problem or something that is regulated in matrices of intelligibility (that need deconstructing and to be 'queeried') (cf. Milani above). However, Oostendorp and Bylund ask us to consider instead the possibility that "linguistic diversity and multilingualism can have positive effects in the context of HIV/Aids communication" by helping to unlock the benefits of attention to the local. Empathy, the intellectual and emotional participation in another person's experience, has been shown to correlate positively with the multilingualism of the individual - perhaps another way of saying that being part of diversity promotes empathy. Turning to multilingualism to enhance empathy is also well in keeping with Chimbutane's insights that using multilingual resources enhances local relevance. Antia and Razum suggest how messages can be better designed in both Germany and Nigeria, by highlighting risk in German context and by offering empowering options (e.g. embedding messages of safe sex) in new structures that service 'agency' in the Nigerian context. Chimbutane's paper also shows the complexities of sexuality in impacting on HIV/AIDS, and how to accept traditional practices, but make them safer (cf. Luphondo and Stroud, of creating alternatives subjectivities that can address the framing conditions of life and sexuality in African context).

Ultimately, the papers all touch on how best to engage with the voices and agencies of the very diverse communities we all increasingly share in. Diversity and cohesion are intrinsic and vulnerable features of a 'moral' society (Heugh in press). Sustainable management of diversity, including stability, heritage and cohesion, is made difficult by the fact that the residues of nineteenth-century concepts of the nation state and ethnolinguistic affiliations no longer offer satisfactory categorizations of social structure, nor capture how individuals and groups perceive themselves (Giddens 1990). The two short research reports that conclude this issue deal precisely with notions of citizenship, specifically health citizenship (Thutloa and Stroud), and inclusive and comprehensive strategies for wide mobilization of stakeholders (Sobane). In fact, we require a notion of citizenship that emphasizes 'agency' and citizenship as "a capacity to act" that is not determined or produced by any one social identity or political alignment but in the multiplicity of relations through which civic associations and attachments are woven" (Rose 2000).

\section{References}

Blommaert, J. and A. Backus. 2011. Repertoires revisited: 'Knowing language' in superdiversity. Working Papers in Urban Language and Literacies 67.

Blommaert, J. and J. Verschueren. 1998. Debating Diversity: Analysing the Discourse of Tolerance. London: Routledge.

Giddens, A. 1990. The consequences of modernity. Cambridge: Polity.

Heugh, K. (in press). Mobility, migration, sustainability. International Journal of Languages.

Higgins, C. and B. Norton. 2010. Language and HIV/AIDS. Clevedon, UK: Multilingual Matters.

Irvine, J. T. and S. Gal. 2000 Language ideology and linguistic differentiation. In P. V. Kroskrity (ed.) Regimes of Language: Ideologies, Polities, and Identities. Santa Fe: School of American Research Press. 
Jagose, A. 1996. Queer Theory: An Introduction. New York: NYU Press.

Passador, L.H. 2009. "Tradition", person, gender, and STD/HIV/AIDS in Southern Mozambique. Forum 25(3): 687-693.

Rose, N. 2000. Governing cities, governing citizens. In E. Isin (ed.) Democracy Citizenship and the Global City. London: Routledge

Stroud, C. and L. Wee. 2006. Style, identity and English language literacy. Linguistics and Education 16: 319-37.

Stroud, C. and L. Wee. 2007. Language policy and linguistic markets in Singapore. Sociolinguistic Studies 1(2): 197-216.

Stroud, C. and L. Wee. 2012. Style, Identity and Literacy: English in Singapore. Bristol: Multilingual Matters

Vertovec, S. 2007. Super-diversity and its implications. Ethnic and Racial Studies 30(6): 1024-1054.

Vertovec, S. 2010. Towards post-multiculturalism? Changing communities, contexts and conditions of diversity. International Social Science Journal 199: 83-95.

Yep, G. 2003. The violence of heteronormativity in communication studies: notes on injury, healing and queer world making. Journal of Homosexuality 45(2-4): 11-59. 